\title{
Stevens-Johnson Syndrome and Toxic Epidermal Necrolysis: A Retrospective Analysis of 23 Patients
}

Başak Yalıcı-Armağan', [MD]

ORCID: 0000-0001-9745-1331

Caner Demircan'2, [MD]

ORCID: 0000-0002-7432-5815

Neslihan Akdoğan ${ }^{3},[M D]$

ORCID: 0000-0002-1137-5399

Duygu Gülseren ${ }^{4}$, [MD]

ORCID: 0000-0003-1602-726X

Sibel Doğan-Günaydın ${ }^{4},[\mathrm{MD}]$

ORCID: 0000-0002-5383-6886

Gonca Elçin ${ }^{5}$ [MD]

ORCID: 0000-0001-6292-7272

Ayşen Karaduman 5 , [MD]

ORCID: 0000-0002-4056-6303

Zehra Nilgün Atakan ${ }^{6}$, [MD]

ORCID: 0000-0002-8790-1607

Sibel Ersoy-Evans ${ }^{5}$, [MD]

ORCID: 0000-0002-5040-2476

'Department of Dermatology and Venereology, Hacettepe University, Faculty of Medicine, Ankara.

${ }^{2}$ Department of Dermatology and Venereology, Medical Park Hospital, Ankara.

${ }^{3}$ Department of Dermatology and Venereology, Hacettepe University, Faculty of Medicine, Ankara.

${ }^{4}$ Department of Dermatology and Venereology, Hacettepe University, Faculty of Medicine, Ankara.

${ }^{5}$ Dermatology and Venereology, Hacettepe University, Faculty of Medicine, Ankara.

${ }^{6}$ Dermatology and Venereology, Private Clinic, Ankara.

Corresponding Author: Başak Yalıcı-Armağan

Department of Dermatology and Venereology,

Hacettepe University, Faculty of Medicine, Ankara,

Turkey.

E-mail: basakarmagan@gmail.com

https://doi.org/10.32552/2021.ActaMedica.660

\section{n ABSTRACT Con}

Objective: Stevens-Johnson syndrome (SJS) and toxic epidermal necrolysis (TEN) are dermatologic emergencies. There is a lack of consensus regarding appropriate management of SJS/TEN. The aim of this study was to evaluate demographic and clinical features, management and outcomes of SJS/TEN patients.

Materials and Methods: The data of patients who were $\geq 18$ years old and hospitalized with the diagnosis of SJS, SJS-TEN overlap and TEN at Hacettepe University, between 1992 and 2018 were analyzed retrospectively. Patient demographics, medications, time between the first causative drug intake and the onset of symptoms, mucous membrane involvement, treatment modalities including supportive measures, intravenous immunoglobulin, cyclosporine and systemic corticosteroids, duration of hospitalization, and mortality outcomes were recorded from patient charts.

Results: A total of 23 patients (11 men; 12 women) with a mean age of $46.4 \pm 19.5$ years were included in the study. Twelve patients $(52.2 \%)$ had SJS, 8 patients (34.8\%) had SJS/TEN overlap and 3 patients (13\%) had TEN. Mean duration of hospitalization was $12.6 \pm 6.5$ days. Twenty-two patients (95.7\%) were attributed to a specific medication, whereas triggering factor was not detected in 1 patient (4.3\%). More than one drug was responsible for 9 patients, including antimicrobials $(n=11,47.8 \%)$, anticonvulsants $(n=8,34.8 \%)$ and/or non-steroid antiinflammatory drugs $(n=5,21.7 \%)$. The median time between the intake of medication and the onset of symptoms was 20 days (IQR 5.5-30). Sixteen patients used systemic corticosteroids (69.6\%) and 4 patients used intravenous immunoglobulin (17.4\%) whereas 3 patients used systemic corticosteroids in combination with intravenous immunoglobulin $(n=2$, $8.7 \%)$ or cyclosporin $(n=1,4.3 \%)$. The median time between the onset of symptoms and the onset of the rash to the treatment was 5 days (IQR 3-7). Mortality was not observed in our cohort.

Conclusion: SJS/TEN was most commonly developed because of drugs, mainly antimicrobials, anticonvulsants and/or non-steroid anti-inflammatory drugs. The absence of mortality in our cohort was considered to be associated with younger age, low rate of TEN, prompt initiation of treatment (mainly corticosteroids) following the rapid discontinuation of the suspected drug.

Keywords: Stevens-Johnson syndrome, toxic epidermal necrolysis, treatment

Received: 2 August 2021, Accepted: 5 September 2021,

Published online: 20 September 2021 


\section{INTRODUCTION}

Stevens-Johnson syndrome (SJS) and toxic epidermal necrolysis (TEN) are rare, acute, lifethreatening adverse mucocutaneous reactions characterized by blistering and erosions of skin and mucous membranes. SJS and TEN are considered to be two variants of the same pathological process that differ depending on the extent of body surface area (BSA) of skin detachment [1]. The skin detachment of SJS, SJS-TEN overlap and TEN is defined as less than $10 \%, 10-30 \%$ and $30 \%$ of BSA, respectively. SJS/TEN usually present with a variety of skin lesions, including patches, atypical targetoid macules, and erythematous or violaceous macules. Mucocutaneous involvement is the characteristic feature of SJS/TEN and the oral mucosa is more commonly involved than the ocular, genital or anal mucosa.

Drugs are the most common cause of SJS/TEN, whereas a minority of cases can be attributed to other exposures, including infection [2]. Antimicrobials, antiepileptics, allopurinol, and nonsteroidal anti-inflammatory medications (NSAIDs) are the most common cause. Typically, the culprit drug has been initiated within the 1-3 weeks prior to the onset of signs and symptoms. Risk factors for the development of SJS/TEN have been described as slow acetylator genotypes who metabolize drugs at a decreased rate, immunosuppression (e.g. HIV infection, lymphoma), concomitant administration of radiotherapy and anticonvulsants and having specific human leukocyte antigen (HLA) alleles.

Although SJS and TEN are rare reactions, they are associated with a high morbidity and mortality rate [3]. SCORTEN criteria were proposed to predict mortality rate in these patients based on 7 independent risk factors including age, malignancy, tachycardia, degree of epidermal detachment at presentation, and serum values of urea, glucose, and bicarbonate with probability of mortality rate varying from $3.2 \%$ to $90 \%$ [4]. Early recognition and prompt management can be life-saving in these reactions [3]. However, there is no established standardized therapeutic option for SJS/TEN due to the rarity and severity of the disease [5] The aim of this study was to evaluate demographic and clinical features, management and outcomes of SJS, SJS-TEN overlap and TEN in our hospitalized patient group.

\section{MATERIALS AND METHODS}

We retrospectively analyzed the data of patients who were $\geq 18$ years old and hospitalized with the diagnosis of SJS, SJS-TEN overlap and TEN at Hacettepe University between January 1, 1992, and January 1, 2018.

Data collected from the reviewed charts included patient demographics (age, sex), relevant past medical history, antecedent use of medications, time between the first causative drug intake and the onset of symptoms, duration of hospitalization, mucous membrane involvement, extent of BSA detachment at admission, treatment modalities including supportive measures, intravenous immunoglobulin (IVIg), cyclosporine and systemic corticosteroids, and mortality outcomes.

\section{Statistical Analysis}

Statistical analysis was performed using IBM SPSS Statistics for Windows v.22 (IBM Corp., Armonk, NY). Continuous variables are presented as mean \pm standard deviation (SD) and median (interquartile range). The Shapiro-Wilk test was used to determine the normality of the distribution of numeric variables. Patient demographic features were analyzed using descriptive statistics. Categorical variables are shown as frequency and percentage.

\section{RESULTS}

\section{Demographic and Clinical Findings}

A total of 23 patients (11 men; 12 women) were enrolled in this study. Mean age was $46.4 \pm 19.5$ years. In total, 12 patients (52.2\%) had SJS, 8 patients (34.8\%) had SJS/TEN overlap and 3 patients (13\%) had TEN. Cutaneous rash was present in 21 patients (91.3\%) of whom 10 (47.6\%) were in atypical macular targetoid appearance. Oral, ocular, genital and nasal mucosa were involved in 18 (78.3\%), 14 $(60.9 \%), 4(17.4 \%)$ and $3(13 \%)$ patients, respectively.

\section{Triggering Factors}

In total, 22 patients (95.7\%) were attributed to a specific medication and triggering factor was not detected in 1 patient (4.3\%). More than one drug 
was recorded to be responsible for 9 patients. Antimicrobials $(n=11,47.8 \%)$, anticonvulsants $(n=8,34.8 \%)$ and/or NSAIDs $(n=5,21.7 \%)$ were responsible in 17 patients (73.9\%). Systemic corticosteroids (deflazacort), antimetabolite drugs (capesitabin) and amifostine were responsible for 2, 2 and 1 patients, respectively. Median time between the intake of medication and the onset of symptoms was 20 days (IQR 5.5-30).

\section{Treatment protocol}

Sixteen patients used systemic corticosteroids (69.6\%) and 4 patients used IVlg (17.4\%) whereas 3 patients used systemic corticosteroids in combination with IVIg $(n=2,8.7 \%)$ or cyclosporin $(n=1,4.3 \%)$. Systemic corticosteroids were recommended in 11 of 12 patients with SJS, whereas in 5 of 11 patients with SJS/TEN or TEN with a total dose of 0.5 to $1 \mathrm{mg} / \mathrm{kg}$ per day. IVlg and corticosteroids in combination with IVIg or cyclosporin were recommended in 1 of 12 patients with SJS and 6 of 11 patients with SJS/TEN or TEN. The median time between the onset of the rash and the treatment was 5 days (IQR 3-7).

Supportive measures and wound care agents including oral antifungal mouthwash, oral anesthetic mouthwash, topical corticosteroid ointment, topical antibiotic ointment, lubricant eye drop and systemic antihistamines were used in 10 (43.4\%), 5 (21.7\%), 8 (34.8\%), 7 (30.4\%), 5 (21.7\%) and 7 (30.4\%) patients, respectively.

Mean duration of hospitalization was $12.6 \pm 6.5$ days. Mortality was not observed in our cohort.

\section{DISCUSSION}

SJS/TEN is one of the few dermatological diseases that constitute a medical emergency. The overall mortality rate ranges from approximately $5-10 \%$ in SJS and $30-50 \%$ in TEN [6]. However, ideal therapy remains a matter of debate mainly due to the disease nature. We conducted a retrospective study to analyze the clinical characteristics of 23 patients who were treated at a tertiary hospital in Turkey. SJS/TEN was most commonly developed due to drugs, mainly antimicrobials, anticonvulsants and/or NSAIDs in our cohort. The majority of our patients (87\%) consisted of SJS or SJS/TEN overlap and only $13 \%$ had TEN. The absence of mortality in our cohort was considered to be associated with younger age range of our patients, low rate of TEN, prompt initiation of treatment (mainly corticosteroids) following the rapid discontinuation of the suspected drug.

SJS/TEN is almost exclusively attributed to drugs; however, infections by viruses, bacteria, and mycobacteria could trigger the development of SJS/ TEN. In a large retrospective cohort from the United States, $89.7 \%$ of cases were attributed to a specific medication, whereas $3.4 \%$ and $6.9 \%$ were classified as due to an infectious trigger and unknown cause, respectively [2]. The most frequently implicated drugs with SJS/TEN primarily consist of allopurinol, antibiotics, NSAIDs, and aromatic anticonvulsants which vary according to the population [7]. In Europe, allopurinol and in Africa sulfonamide antibiotics are the most common agents causing SJS/TEN [8]. A strong association has been defined between HLA-B*58:01 allele and allopurinol induced SJS/TEN development in Han Chinese population [9]. In the present study, $95.7 \%$ of the patients have been associated with a drug of which antibiotics were most common responsible drug. The absence of allopurinol-induced cases may be related to genetic insusceptibility of our population. Because infection tests, including Herpes simplex virus tests, Mycoplasma pneumonia and Human immunodeficiency virus were not performed in the present case of unknown trigger, we could not clearly exclude the possibility of an infectious origin.

Interestingly, corticosteroids, which are potent anti-inflammatory agents used in the treatment of various inflammatory diseases, can induce SJS/TEN [10]. Deflazacort induced drug reactions, including maculopapular rash, acute exanthematous pustulosis, erythema multiforme and SJS/TEN have been reported previously [11]-[13]. Similarly, deflazacort was responsible for SJS development in $8.9 \%(n=2)$ of our patients. Systemic prednisolone treatment was used successfully in these patients. It is critical for clinicians to be aware that SJS/TEN can also be triggered by corticosteroids in order to develop an appropriate treatment approach.

Identification of the responsible drug is an important and difficult task, but it should be among the first priorities. SJS and TEN usually occur 7-21 days after initiation of the causative drug if it is the first exposure to the drug, but can occur within 2 
days with re-exposure to a drug that previously caused SJS or TEN. , However, it may be longer than 30 weeks in low-risk drugs [14]. Compatible with the literature data, median time between drug intake and onset of rash was 20 days in our study. To chart a timeline for each exposed drug, regardless of the route of administration or frequency of usage, is crucial in order to visualize the chronology and find out the suspected drugs, since the main goal of the treatment is to withdraw of the responsible drug.

Systemic corticosteroids are most commonly used agents for SJS/TEN worldwide. However, the efficacy of systemic corticosteroids for SJS/TEN is still controversial [5]. Early observational studies suggested that patients treated with corticosteroids had significantly higher rates of infection and overall complications, including higher rates of mortality [15], [16]. A RegiSCAR (International Registry of Severe Cutaneous Adverse Reactions to drugs) study showed no survival benefit for patients treated with corticosteroids compared to those treated with supportive care only [17]. On the other hand, the beneficial effect of short-term corticosteroids has been also reported in some studies, especially with early administration and pulse therapy [18]-[20]. Systemic corticosteroids were the most common drugs used in our study and no mortality was observed in our cohort. Although our patient group is younger and the number of severe patients is limited, our results may suggest a potential benefit of medium-dose corticosteroids for SJS/TEN management. We think that early initiation of treatment also contributes to the response.

Besidescorticosteroids, variousimmunomodulating agents including cyclosporine, IVIg, plasmapheresis, tumor necrosis factor a inhibitors (e.g. etanercept and infliximab), thalidomide and cyclophosphamide have been proposed to treat SJS/TEN but their efficacy remains unclear, yet. A phase II trial by Valeyrie-Allanore et al. [21] found that among 45 patients treated with cyclosporine, the death rate and progression of detachment were lower than expected. A retrospective study of 64 cases conducted by Kirchoff et al. [22] reported that cyclosporine was superior to IVIg in terms of mortality. A study by Wang et al. [23] found etanercept was superior than systemic corticosteroids in the treatment of SJS/TEN. A recent systematic review and meta-analysis of 67 studies and 2079 patients showed that combination therapy with corticosteroids and IVIg could reduce the risk of mortality in patients with SJS/TEN; while cyclosporine and etanercept are promising therapies but more studies are needed to provide clearer evidence [24]. In our study, IVIg was used with or without corticosteroids in approximately one fourth of the patients successfully. However, cyclosporine was used in combination with corticosteroids in only 1 patient and etanercept was not used in any patient due to the relatively recent demonstration of their effectiveness. Although IVIg and cyclosporine were recommended for patients with more severe detachment (SJS/TEN overlap and TEN), the absence of mortality in our study was compatible with up-to-date data [19].

The limitations of this study include its retrospective nature, not predicting the prognosis with SCORTEN and the heterogeneous treatment protocol, which makes it difficult to compare treatment responses.

Antibiotics, anticonvulsants and/or NSAIDs were most common drugs associated with SJS/TEN in our population. Besides prompt cessation of suspected drug, early initiation of medium dose corticosteroids and/or IVIG seem to have a positive effect on the management of SJS/TEN.

\section{CONFLICT OF INTEREST STATEMENT}

We declare that there is no conflict of interest of all authors in this work. No financial support was provided for the conduct, preparation, collection, analysis, interpretation and writing of the report. 
[1] Bastuji-Garin S, Rzany B, Stern RS et al. Clinical classification of cases of toxic epidermal necrolysis, Stevens-Johnson syndrome, and erythema multiforme. Arch Dermatol. 1993;129(1):92-6.

[2] Micheletti RG, Chiesa-Fuxench Z, Noe MH et al. StevensJohnson Syndrome/Toxic Epidermal Necrolysis: A Multicenter Retrospective Study of 377 Adult Patients from the United States. J. Invest Dermatol. 2018;138(11):23152321.

[3] Lee HY, Walsh SA, Creamer D. Long-term complications of Stevens-Johnson syndrome/toxic epidermal necrolysis (SJS/TEN): the spectrum of chronic problems in patients who survive an episode of SJS/TEN necessitates multidisciplinary follow-up. $\mathrm{Br} J$ Dermatol. 2017;177(4):924-935.

[4] Bastuji-Garin S., Fouchard N, Bertocchi M. et al. SCORTEN: A Severity-of-Illness Score for Toxic Epidermal Necrolysis. J Invest Dermatol 2000;115:149-153.

[5] Schneck J, Fagot J.P., Sekula P. et al. Effects of treatments on the mortality of Stevens-Johnson syndrome and toxic epidermal necrolysis: A retrospective study on patients included in the prospective EuroSCAR Study. J Am Acad Dermatol 2008;58:33-40.

[6] Chung W.H.,Wang C.W., and Dao R.Let al. Severe cutaneous adverse drug reactions. J Dermatol. 2016;43(7):758-66.

[7] Sassolas B, Haddad C, Mockenhaupt M. et al. ALDEN, an algorithm for assessment of drug causality in stevensjohnson syndrome and toxic epidermal necrolysis: Comparison with case-control analysis. Clin. Pharmacol. Ther. 2010;88(1):60-8.

[8] Dodiuk-Gad R.P., Chung WH, L. Valeyrie-Allanore L. et al. Stevens-Johnson Syndrome and Toxic Epidermal Necrolysis: An Update. Am J Clin Dermatol. 2015;16(6):47593.

[9] Hung SI, Chung WH, Liou LB. et al. HLA-B*5801 allele as a genetic marker for severe cutaneous adverse reactions caused by allopurinol. Proc Natl Acad Sci USA. 2005;102(11):4134-9.

[10] J C Roujeau JC, Kelly JP, Naldi L et al. Medication Use and the Risk of Stevens-Johnson Syndrome or Toxic Epidermal Necrolysis. N Engl J Med. 1995;333(24):1600-7.

[11] Samimi S.S. and Siegfried E. Stevens-Johnson syndrome developing in a girl with systemic lupus erythematosus on high-dose corticosteroid therapy. Pediatr Dermatol. JanFeb 2002;19(1):52-5.

[12] Lee EC, Kim GA, Koo JW. Toxic epidermal necrolysis associated with deflazacort therapy with nephrotic syndrome. Kidney Res Clin Pract. 2014;33(4):222-5.

[13] Trujillo-Trujillo MJ, González-Mancebo E, GandolfoCano M. et al. Deflazacort-induced erythema multiforme exudativum successfully treated with a single dose of etanercept. J Investig Allergol Clin Immunol. 2018;28(5):338-339.
[14] Mockenhaupt M, Viboud C, Dunant A. et al.StevensJohnson syndrome and toxic epidermal necrolysis: Assessment of medication risks with emphasis on recently marketed drugs. The EuroSCAR-study. J Invest Dermatol. 2008;128(1):35-44.

[15] Kim PS, Goldfarbl W, Gaisford JC, et al. Stevens \pm Johnson syndrome and toxic epidermal necrolysis: a pathophysiologic review with recommendations for a treatment protocol. J Burn Care Rehabil. 1983;4:91-100.

[16] Halebian PH, Corder VJ, Madden MR. et al. Improved burn center survival of patients with toxic epidermal necrolysis managed without corticosteroids. Ann Surg. 1986;204:512-513.

[17] Sekula P, Dunant A., Mockenhaupt M. et al.Comprehensive survival analysis of a cohort of patients with StevensJohnson syndrome and toxic epidermal necrolysis. J. Invest Dermatol. 2013;133(5):1197-1204.

[18] Hirahara K.Kano Y. Sato Y et al. Methylprednisolone pulse therapy for Stevens-Johnson syndrome/toxic epidermal necrolysis: Clinical evaluation and analysis of biomarkers. J. Am. Acad. Dermatol.2013;69(3):496-498.

[19] Kardaun SH, and Jonkman MF. Dexamethasone pulse therapy for Stevens-Johnson syndrome/toxic epidermal necrolysis. Acta Derm Venereol. 2007;87(2):144-8.

[20] Roongpisuthipong W., Prompongsa S, Klangjareonchai T.Retrospective analysis of corticosteroid treatment in Stevens-Johnson syndrome and/or toxic epidermal necrolysis over a period of 10 years in Vajira Hospital, Navamindradhiraj University, Bangkok. Dermatol Res Pract. 2014; 2014:237821.

[21] Valeyrie-Allanore L, Wolkenstein P, Brochard L et al. Open trial of ciclosporin treatment for Stevens-Johnson syndrome and toxic epidermal necrolysis. $\mathrm{Br} J$ Dermatol. 2010;163(4):847-53.

[22] Kirchhof MG, Miliszewski MA, Sikora S et al. Retrospective review of Stevens-Johnson syndrome/toxic epidermal necrolysis treatment comparing intravenous immunoglobulin with cyclosporine. J Am Acad Dermatol. 2014;71(5):941-7.

[23] Olteanu C, Shear NH, Burnett M. et al. Retrospective Study of Patients With SJS/TEN Treated at a Tertiary Burn Unit in Canada: Overview of 17 Years of Treatment. J Cutan Med Surg. 2021;25(3):271-280.

[24] Tsai TY, Huang IH, Chao YC et al. Treating toxic epidermal necrolysis with systemic immunomodulating therapies: A systematic review and network meta-analysis. J Am Acad Dermatol. 2021;84(2):390-397. 